\title{
Data quality of drug-resistant tuberculosis and antiretroviral therapy electronic registers in South Africa
}

\author{
Lise Jamieson ${ }^{1 *}$ D, Denise Evans ${ }^{1}$, Rebecca Berhanu ${ }^{1,2}$, Nazir Ismail ${ }^{3,4}$, Samantha Aucock ${ }^{5}$, Kristina Wallengren ${ }^{5}$ and \\ Lawrence Long ${ }^{1,2}$
}

\begin{abstract}
Background: To assess the quality and completeness of treatment and outcome data in the electronic tuberculosis (TB) and antiretroviral treatment (ART) registers in drug-resistant (DR-) TB patients at three treatment facilities in South Africa.

Methods: We did a retrospective cohort study using routinely-collected data from DR-TB registers of rifampicin resistant adults ( $\geq 18$ years old), on ART, initiating DR-TB treatment between January 2012 and December 2013. We linked patient information from the DR-TB register to the ART register using patient identifiers and an algorithm based on string edit distance and date of birth. We describe data gaps and discrepancies found.

Results: Overall, 2852 DR-TB patients met our inclusion criteria based on the DR-TB register data, and of these, 1685 (59\%) could be matched to the ART registers. An additional 253 patients from the DR-TB registers were found in the ART registers, having initiated ART, despite the DR-TB register indicating that they were not on ART (or this data was missing). $11 \%$ of matched patients did not have TB treatment status recorded in the ART register despite being recorded as being on TB treatment in the DR-TB register, and 78\% did not have an ART start date recorded in DRTB register despite being on ART treatment as per the ART register. 11\% of matched patients had a death recorded in one register but not the other, and of those with death recorded in both, 15\% of dates differed by $>1$ month.

Conclusions: The underreporting of death and the lack of ART or TB status in the electronic DR-TB and ART registers could negatively impact monitoring efforts by downplaying the state of the TB/HIV epidemic. Improved recording of these data sources, and data integration across systems, could improve the accuracy of reporting for the national HIV/ART and TB programs.
\end{abstract}

Keywords: EDRWeb, Tier, net, Data quality, Routine data

\section{Background}

South Africa has the largest population of patients on antiretroviral therapy (ART) at 4.36 million in 2017 [1]. Approximately 322,000 active tuberculosis (TB) cases and 16,000 new multi-drug resistant (MDR) or rifampicin-resistant (RR-) TB cases are estimated to occur each year [2]. The majority of the population affected by HIV and TB access their treatment through

\footnotetext{
* Correspondence: ljamieson@heroza.org

${ }^{1}$ Health Economics and Epidemiology Research Office (HE2RO), Department of Internal Medicine, School of Clinical Medicine, Faculty of Health Sciences, University of the Witwatersrand, Johannesburg, South Africa

Full list of author information is available at the end of the article
}

the public healthcare system. While health facilities keep their own paper records and registers, they are required to report into the national electronic registers for monitoring of HIV and TB. Two TB registers are widely in use: the national electronic TB register (ETR.Net) data for drug-sensitive $\mathrm{TB}$ and the electronic drug-resistant TB register (EDRWeb) for drug-resistant (DR)-TB. The Three Integrated Electronic Registers (TIER.NET) is a national monitoring/evaluation software system used to capture standardized data of patients on ART [3]. Introduced in 2011, it is used in over 3000 facilities in South Africa.

(c) The Author(s). 2019 Open Access This article is distributed under the terms of the Creative Commons Attribution 4.0 International License (http://creativecommons.org/licenses/by/4.0/), which permits unrestricted use, distribution, and reproduction in any medium, provided you give appropriate credit to the original author(s) and the source, provide a link to the Creative Commons license, and indicate if changes were made. The Creative Commons Public Domain Dedication waiver (http://creativecommons.org/publicdomain/zero/1.0/) applies to the data made available in this article, unless otherwise stated. 
Effective monitoring of the national HIV and TB programmes relies on complete, accurate data. Knowing drug-resistant TB incidence within the HIV programme is critical. Furthermore, HIV infection rates and ART uptake amongst patients with $\mathrm{TB}$ are key indicators of TB and HIV programme integration. Accurate recording and reporting is crucial for programme monitoring, resource allocation, policy making and epidemiological analysis.

Though there was an evaluation of the implementation of TIER.NET [4] there have been no studies which assessed quality and completeness. A few studies have assessed the quality and completeness of the ETR. Net and EDRWeb [5-8]. A study of pediatric TB cases demonstrated that a third of these are never recorded in the ETR. Net [5], and another study found that in EDRWeb, $12 \%$ have missing HIV status and 4\% have incorrect HIV status [8]. Although $90 \%$ of adult patients with TB are recorded in the ETR. Net [7], estimates for accurate reporting of HIV status range between 53.9 and $79 \%[6,7]$ and reporting of ART status is as low as $24 \%$ [7]. In a study comparing ETR. Net to clinic source documents, agreement of ART status and TB treatment outcome was low at 59 and $47 \%$, respectively [6]. A study comparing ETR. Net data to the National Health Laboratory Service data found 20\% of smear positive TB cases were not registered in the ETR. Net and only $69.9 \%$ had patient records at the facilities from where the sample was sent [9].

We linked routinely-collected data from DR-TB and ART registers using patient identifiers (name, surname, date of birth, national identification number, if available). This paper aims to describe the methods used to match patients across routine data platforms, and data gaps and discrepancies found.

\section{Methods}

\section{Data sources}

In 2016/17 we extracted EDRWeb and TIER.NET data for two South African healthcare facilities. In addition to EDRWeb and TIER.NET, we included two additional sources of TB and HIV data, as these systems were used at a third healthcare facility we evaluated: the Focal Point Information System (FIS) is an electronic TB patient record system, allowing for multi-point data entry and is being used at three DR-TB treatment sites in South Africa. TherapyEdge-HIV $\mathrm{V}^{\mathrm{mm}}$ (TE) is an electronic medical database system designed for HIV patient management currently used at six sites in South Africa. FIS and TE are more comprehensive than EDRWeb and TIER.NET in terms of variables collected. These systems were implemented at various time points at each site ranging from 2004 to 2013; at the time of data collection all the sites had a minimum of 3 years' experience with their respective systems. In most instances data entry occurred from dedicated data staff at the site, but could also have been entered by other administrative or clinical staff. Some systems were donor funded and therfore had external support to implement and manage them (i.e. TE and FIS). TIER.NET and EDRWeb are government-mandated systems and implementation and management is through the South African National Department of Health (NDOH). To our knowledge there was no external validation or oversight of these electronic registers.

As this study could not have been done without knowing patient identifiers, a number of steps were taken to mitigate risks to patient confidentiality: 1) datasets were collected directly from each facility, password protected and transported on an encrypted hard-drive, 2) access to the data was limited to a single person who performed all analyses, 3) analytic datasets beyond the matching algorithm were de-identified for subsequent analyses.

\section{Study sites}

The three facilities were based in the Gauteng and KwaZulu-Natal provinces. One is an outpatient NonGovernmental Organization (NGO)-supported DR-TB clinic at a public academic hospital. Another facility is a MDR-TB hospital providing inpatient and outpatient care. The third facility is an outpatient DR-TB centre. To maintain anonymity, we labelled them Site 1, 2 and 3. Site 1 uses the FIS and TE systems, while sites 2 and 3 use EDRWeb and TIER.NET.

Inclusion criteria for the study were: adults (aged 18+ years), laboratory diagnosis of rifampicin (RIF) resistant $\mathrm{TB}$ and initiated second-line $\mathrm{TB}$ treatment between January 2012-December 2013. HIV and ART status was primarily determined from either DR-TB or ART register. In both EDRWeb and FIS, ART status was recorded as a binary yes/no variable for ART started or as an ART start date, and either was used to identify ART status. Then, since the DR-TB registers may have incomplete HIV status and/or ART initiation information, we matched DR-TB patients with negative/missing HIV status, or HIV positive and not on ART (or whose ART status was unknown/missing) in the DR-TB register, to the ART register, in order to confirm HIV and/or ART status. Once HIV and ART status had been confirmed, subsequent analyses were restricted to patients who were HIV positive and on ART.

\section{Matching algorithm}

We matched patients from the DR-TB register to their ART record by first merging those where the first letter of either the name or surname matched, and then using a generalized Levenshtein edit distance [10] to match names and surnames between the two datasets. The edit distance is a measure of similarity between two strings. Second or third names were also considered, if recorded. 
SAS version 9.3 was used for analysis, and the built-in function, COMPGED [11] was used.

Patients were marked as a match if their name in the one dataset matched their name in the other dataset with an edit distance of 100 or less $[12,13]$, and their birthdate matched. For patients with a missing date of birth, we matched on patient age and gender, alongside the names, allowing for a 1-year difference. For a birthdate match, we allowed for the year to shift up or down by 1 year, and we allowed for the month of birth to shift up or down by 1 month. We also allowed for the birthdate day and month to be erroneously switched in the datasets. Patients were considered a match if their South African identification number was an exact match. Then, in the case of an exact match of the surname and date of births between the two datasets, we were more lenient on the edit distance for the names (also for first, second or third name matches) by allowing for a score of 500 or less.

\section{Validation of matching}

A random sample of $10 \%$ of each healthcare facility's DR-TB patients meeting our inclusion criteria was selected. A manual matching process, considered the 'gold standard', was followed looking for patients in the corresponding ART register. We calculated sensitivity, specificity, positive predictive value (PPV) and negative predictive value (NPV).

\section{Statistical analysis}

We compared demographics, TB characteristics and outcomes between those patients we could match and those we could not. We describe completeness and discrepancies of common variables in both ART and DR-TB registers. For reporting of death outcomes and ART start dates we calculated a Kappa statistic to measure agreement between the two data sources. The severity of date discrepancies was assessed by calculating the proportion of dates differing by $>1$ month.

\section{Results}

Overall, 2852 DR-TB adult patients, HIV+ on ART, initiated TB treatment between January 2012-December 2013 based on the DR-TB register data, and of these, 1685 (59\%) could be matched to the ART registers. Of the 1526 patients who were reportedly not on ART (or the data was missing), 253 patients were found in the ART registers, having initiated ART. This brought our total matched up to 1938 (62\%) and a total of 3105 DRTB patients who were included in the subsequent analyses. While the rate of matching was high in Sites 1 and 2 , with 76 and $80 \%$, respectively, we could only match $59 \%$ of patients at Site 3. There were no substantial differences in age, gender, new treatment status, extrapulmonary $\mathrm{TB}$ or $\mathrm{TB}$ outcome between those matched compared to those we couldn't match to the ART register (Table 1), though the number of characteristics we could assess was limited.

In order to validate our matching algorithm, we randomly sampled 171 patients and compared the algorithm to our "gold standard" of manual matching. We found our algorithm to have a sensitivity of $87 \%$, a specificity of $99 \%$, a PPV of $98 \%$ and a NPV of $94 \%$. The specificity was reduced by $1 \%$ and PPV by $2 \%$ due to an error in misclassification for one patient in the manual matching process.

Discrepancies between ART and DR-TB registers are presented in Table 2. Overall, $8.1 \%$ of patients had a discrepant date of birth, however the matching algorithm was more lenient on these if the name and surname were matched already; another $2.5 \%$ of patients had a date of birth missing in either register so these patients would have been matched on age, alongside gender and names. All patients in Site 1 had their HIV status recorded as HIV positive in the DR-TB register, but 6.2 and $4.5 \%$ of patients in Sites 2 and 3, respectively, had either a negative, unknown or missing HIV status in the DR-TB register, despite being initiated on ART. Overall, $8.7 \%$ of patients had no record of TB treatment (e.g. missing) in the ART register data and $2.5 \%$ of patients were recorded as not being on TB treatment (e.g. TB treatment incorrectly recorded as "No") in the ART register record. Of the 217 patients whose TB treatment status was incorrectly recorded in the ART register, $82 \%$ had initiated ART prior to TB treatment and thus this is likely due to the records not being updated. However, of all patients who had initiated ART prior to TB treatment $(61 \%, n=1187)$, majority $(85 \%)$ had TB treatment status recorded correctly, demonstrating that most records had been updated in real time.

Majority (87\%) had ART status correctly recorded in the DR-TB register. While ART start date was reported in both ART and DR-TB registers for most patients at Site 1 (81.2\%), 67.8\% were discrepant, and three quarters of those discrepant differed by $>1$ month. At Site 1, 12\% of patients had a missing ART start date in the ART register, though the data indicated they initiated treatment. Most patients at Sites 2 and 3 did not have ART start date recorded in the DR-TB register (99.7 and $80.5 \%$, respectively). Of the 280 patients at Site 3 that did have ART start date recorded in both registers, $15.4 \%$ had discrepant dates with $68.7 \%$ of ART start dates differing by $>1$ month. Overall there was poor agreement of the reporting of the ART start date between the ART and DR-TB registers (Kappa $=0.001$ ). Sites 2 and 3 did not have ART regimen information recorded in the DR-TB register so we could not compare between the two datasets. At Site 1, where ART regimen was recorded in both DR-TB and ART registers for 
Table 1 Differences between matched and unmatched cohort of DR-TB patients initiated on antiretroviral therapy

\begin{tabular}{|c|c|c|c|}
\hline & $\begin{array}{l}\text { All DR-TB/ART patients } \\
(n=3105)\end{array}$ & Not matched to ART data $(n=1167)$ & $\begin{array}{l}\text { Matched to ART data } \\
(n=1938)\end{array}$ \\
\hline \multicolumn{4}{|l|}{ Site, n (\%) } \\
\hline Site 1 (FIS/TE) & $186(6.0 \%)$ & 37 (3.2\%) & $149(7.7 \%)$ \\
\hline Site 2 (EDRWeb/TIER.NET) & $449(14.5 \%)$ & 109 (9.4\%) & $340(17.5 \%)$ \\
\hline Site 3 (EDRWeb/TIER.NET) & $2470(79.5 \%)$ & $1021(87.5 \%)$ & 1449 (74.8\%) \\
\hline \multicolumn{4}{|l|}{ Gender, n (\%) } \\
\hline Female & $1615(52.1 \%)$ & $614(52.7 \%)$ & $1001(51.7 \%)$ \\
\hline Male & 1489 (48.0\%) & $553(47.4 \%)$ & $936(48.3 \%)$ \\
\hline Age, median (IQR) & $35(29-41)$ & $35(30-41)$ & $34(29-41)$ \\
\hline \multicolumn{4}{|l|}{ Patient category } \\
\hline New & 1196 (38.5\%) & $430(36.8 \%)$ & $766(39.5 \%)$ \\
\hline Treatment after failure & 1201 (38.7\%) & $465(39.8 \%)$ & $736(38.0 \%)$ \\
\hline Relapse & 495 (15.9\%) & $198(17.0 \%)$ & $297(15.3 \%)$ \\
\hline Treatment after loss to follow-up & $183(5.9 \%)$ & $64(5.5 \%)$ & 119 (6.1\%) \\
\hline Transfer-in & $1(<0.1 \%)$ & $1(0.1 \%)$ & $0(0.0 \%)$ \\
\hline Other & $7(0.2 \%)$ & $4(0.3 \%)$ & $3(0.2 \%)$ \\
\hline Unknown & $22(0.7 \%)$ & $5(0.4 \%)$ & $17(0.9 \%)$ \\
\hline \multicolumn{4}{|l|}{ TB type } \\
\hline Extra-pulmonary TB & $82(2.6 \%)$ & $21(1.7 \%)$ & $61(3.1 \%)$ \\
\hline Pulmonary TB & 2672 (86.1\%) & 1045 (89.6\%) & $1627(84.0 \%)$ \\
\hline Missing & $351(11.3 \%)$ & $101(8.7 \%)$ & $250(12.9 \%)$ \\
\hline \multicolumn{4}{|l|}{ TB outcome } \\
\hline Cure & 1038 (33.4\%) & 431 (36.9\%) & $607(31.3 \%)$ \\
\hline Completed treatment & $661(21.3 \%)$ & $210(18.0 \%)$ & $451(23.3 \%)$ \\
\hline Death & $557(17.9 \%)$ & $208(17.8 \%)$ & $349(18.0 \%)$ \\
\hline Treatment failure & $72(2.3 \%)$ & $19(1.6 \%)$ & $53(2.7 \%)$ \\
\hline Lost to follow-up & $689(22.2 \%)$ & $276(23.7 \%)$ & $413(21.3 \%)$ \\
\hline Transferred out & $30(1.0 \%)$ & $13(1.1 \%)$ & $17(0.9 \%)$ \\
\hline Still on treatment & $17(0.5 \%)$ & $2(0.2 \%)$ & $15(0.8 \%)$ \\
\hline Other & $1(0.0 \%)$ & $0(0.0 \%)$ & $1(0.1 \%)$ \\
\hline Not specified & $27(0.9 \%)$ & $3(0.3 \%)$ & $24(1.2 \%)$ \\
\hline
\end{tabular}

Abbreviations: DR-TB Drug resistant tuberculosis, ART Antiretroviral therapy, FIS Focal Point Information System, TE TherapyEdge-HIV ${ }^{\mathrm{TM}}$, EDRWeb Electronic Drug Register Web, TIER.NET The Three Integrated Electronic Registers, IQR Interquartile range

$44.3 \%$ of patients, it was in agreement for majority of them $(86.4 \%)$. However, $11.4 \%$ of patients at Site 1 did not have ART regimen recorded in the ART register.

Overall, 7.3\% of patients had a death reported in the DRTB register but not the ART register. Of these $80.9 \%$ were reported in the ART register as a lost to follow-up, 14.9\% as transferred out, and $2.8 \%$ of patients had no outcome reported. While these may be explained by the timing of when the information gets recorded, a large proportion of these deaths (45.4\%) were reported as deaths in the DR-TB register less than 3 months after the non-death outcome in the ART register and $36.9 \%$ had dates which occurred prior to the non-death outcome date in the ART register.
Overall, 3.5\% had a death reported in the ART register but not the DR-TB register. Of these, $56.7 \%$ were reported as lost to follow-up in the DR-TB register, $16.4 \%$ had no outcome reported, and one patient was reportedly transferred out. The remaining patients had TB treatment-specific outcomes reported: $11.9 \%$ cured, $9.0 \%$ completed treatment, $3.0 \%$ still on treatment and one patient had failed TB treatment. Of the 67 patients with death reported in the ART register and not the DR-TB register, 29.9\% were reported as deaths in the ART register $<3$ months after the non-death outcome in the DRTB register and $34.3 \%$ had their death dates prior to the non-death outcome date in the DR-TB register. Overall 
Table 2 Discrepancies in data found between ART and DR-TB registers in patients matched between data sources

\begin{tabular}{|c|c|c|c|c|}
\hline & $\begin{array}{l}\text { Site } 1 \\
\text { (FIS/TE) } \\
(n=149)\end{array}$ & $\begin{array}{l}\text { Site } 2 \\
\text { (EDRWeb/ } \\
\text { TIER.NET) } \\
(n=340)\end{array}$ & $\begin{array}{l}\text { Site } 3 \\
\text { (EDRWeb/ } \\
\text { TIER.NET) } \\
(n=1449)\end{array}$ & $\begin{array}{l}\text { Total } \\
(n=1938)\end{array}$ \\
\hline \multicolumn{5}{|l|}{ Reporting of date of birth } \\
\hline Agree & $92.6 \%(138)$ & $92.7 \%(315)$ & $88.3 \%(1279)$ & $89.4 \%(1732)$ \\
\hline Discrepant between DR-TB and ART register & $7.4 \%(11)$ & $7.4 \%(25)$ & $8.4 \%(121)$ & $8.1 \%(157)$ \\
\hline Missing in ART register & $0.0 \%(0)$ & $0.0 \%(0)$ & $0.1 \%(2)$ & $0.1 \%(2)$ \\
\hline Missing in DR-TB register & $0.0 \%(0)$ & $0.0 \%(0)$ & $3.2 \%(47)$ & $2.4 \%(47)$ \\
\hline \multicolumn{5}{|l|}{ DR-TB treatment recorded in ART register } \\
\hline Not recorded/missing & $3.4 \%(5)$ & $42.4 \%(144)$ & $1.4 \%(20)$ & $8.7 \%(169)$ \\
\hline Has not started TB treatment & $0.0 \%(0)$ & $2.7 \%(9)$ & $2.7 \%(39)$ & $2.5 \%(48)$ \\
\hline Started TB treatment & $96.6 \%(144)$ & $55.0 \%(187)$ & $95.9 \%(1390)$ & $88.8 \%(1721)$ \\
\hline \multicolumn{5}{|l|}{ HIV status reported in DR-TB register } \\
\hline Positive & $100 \%(149)$ & $93.8 \%(319)$ & $95.6 \%(1385)$ & $95.6 \%(1853)$ \\
\hline Negative & $0.0 \%(0)$ & $5.6 \%(19)$ & $2.6 \%(37)$ & $2.9 \%(56)$ \\
\hline Unknown or missing & $0.0 \%(0)$ & $0.6 \%(2)$ & $1.9 \%(27)$ & $1.5 \%(29)$ \\
\hline \multicolumn{5}{|l|}{ ART status recorded in DR-TB register } \\
\hline Not recorded/missing & $0.0 \%(0)$ & $1.5 \%(5)$ & $3.7 \%(53)$ & $3.0 \%(58)$ \\
\hline Has not started ART & $6.7 \%(10)$ & $5.6 \%(19)$ & $11.7 \%(169)$ & $10.2 \%(198)$ \\
\hline Started ART & $93.3 \%(139)$ & $92.9 \%(316)$ & $84.7 \%(1227)$ & $86.8 \%(1682)$ \\
\hline \multicolumn{5}{|l|}{ Reporting of ART start date } \\
\hline Not recorded in either register & $1.3 \%(2)$ & $0.0 \%(0)$ & $0.1 \%(2)$ & $0.2 \%(4)$ \\
\hline Recorded in ART register, but not DR-TB register & $6.7 \%(10)$ & $99.7 \%(339)$ & $80.5 \%(1167)$ & $78.2 \%(1516)$ \\
\hline Recorded in DR-TB register, but not ART register & $10.7 \%(16)$ & $0.0 \%(0)$ & $0.0 \%(0)$ & $0.1 \%(16)$ \\
\hline Recorded in both registers & $81.2 \%(121)$ & $0.3 \%(1)$ & $19.3 \%(280)$ & $20.7 \%(402)$ \\
\hline ART start dates agree & $\begin{array}{l}32.2 \%(39 / \\
121)\end{array}$ & $0.0 \%(0 / 1)$ & $\begin{array}{l}84.6 \% \text { (237/ } \\
280)\end{array}$ & $\begin{array}{l}68.7 \%(276 / \\
402)\end{array}$ \\
\hline ART start dates differ by $\leq 1$ month & $\begin{array}{l}17.3 \%(21 / \\
121)\end{array}$ & $0.0 \%(0 / 1)$ & $5.4 \%(15 / 280)$ & $9.0 \%(36 / 402)$ \\
\hline ART start dates differ by $>1$ month & $\begin{array}{l}50.4 \%(61 / \\
121)\end{array}$ & $100 \%(1 / 1)$ & $10.0 \%(28 / 280)$ & $22.4 \%(90 / 402)$ \\
\hline \multicolumn{5}{|l|}{ Reporting of ART regimen } \\
\hline Not recorded in either register & $8.1 \%(12)$ & $0.0 \%(0)$ & $0.0 \%(0)$ & $0.6 \%(12)$ \\
\hline Recorded in ART register, but not DR-TB register & $44.3 \%(66)$ & $100 \%(340)$ & $100 \%(1449)$ & $95.7 \%(1855)$ \\
\hline Recorded in DR-TB register, but not ART register & $3.4 \%(5)$ & $0.0 \%(0)$ & $0.0 \%(0)$ & $0.3 \%(5)$ \\
\hline Recorded in both registers & $44.3 \%(66)$ & $0.0 \%(0)$ & $0.0 \%(0)$ & $3.4 \%(66)$ \\
\hline ART regimens agree & $86.4 \%(57 / 66)$ & N/A & N/A & $86.4 \%(57 / 66)$ \\
\hline \multicolumn{5}{|l|}{ Reporting of death and date of death } \\
\hline No deaths in either register & $81.9 \%(122)$ & $84.4 \%(287)$ & $76.8 \%(1113)$ & $78.5 \%(1522)$ \\
\hline Death in ART register, but not DR-TB register & $2.7 \%(4)$ & $1.5 \%(5)$ & $4.0 \%(58)$ & $3.5 \%(67)$ \\
\hline \multicolumn{5}{|l|}{ Reported in DR-TB register as: } \\
\hline Lost to follow-up & 3 & 5 & 30 & 38 \\
\hline Transferred out & 1 & 0 & 0 & 1 \\
\hline Cured & 0 & 0 & 8 & 8 \\
\hline Treatment completed & 0 & 0 & 6 & 6 \\
\hline Still on treatment & 0 & 0 & 2 & 2 \\
\hline
\end{tabular}


Table 2 Discrepancies in data found between ART and DR-TB registers in patients matched between data sources (Continued)

\begin{tabular}{|c|c|c|c|c|}
\hline & $\begin{array}{l}\text { Site } 1 \\
\text { (FIS/TE) } \\
(n=149)\end{array}$ & $\begin{array}{l}\text { Site } 2 \\
\text { (EDRWeb/ } \\
\text { TIER.NET) } \\
(n=340)\end{array}$ & $\begin{array}{l}\text { Site } 3 \\
\text { (EDRWeb/ } \\
\text { TIER.NET) } \\
(n=1449)\end{array}$ & $\begin{array}{l}\text { Total } \\
(n=1938)\end{array}$ \\
\hline Treatment failure & 0 & 0 & 1 & 1 \\
\hline Not specified & 0 & 0 & 11 & 11 \\
\hline Death in DR-TB register, but not ART register & $4.7 \%(7)$ & $2.9 \%(10)$ & $8.6 \%(124)$ & $7.3 \%(141)$ \\
\hline \multicolumn{5}{|l|}{ Reported in ART register as: } \\
\hline Lost to follow-up & 0 & 8 & 106 & 114 \\
\hline Transferred out & 3 & 2 & 16 & 21 \\
\hline Not specified & 4 & 0 & 0 & 4 \\
\hline Death in both registers & $10.7 \%(16)$ & $11.2 \%(38)$ & $10.6 \%(154)$ & $10.7 \%(208)$ \\
\hline Death dates agree & $25.0 \%(4 / 16)$ & $\begin{array}{l}55.3 \%(21 / \\
38)\end{array}$ & $59.1 \%(91 / 154)$ & $\begin{array}{l}55.8 \%(116 / \\
208)\end{array}$ \\
\hline Death dates differ by $\leq 1$ month & $31.3 \%(5 / 16)$ & $\begin{array}{l}39.5 \%(15 / \\
38)\end{array}$ & $26.0 \%(40 / 154)$ & $28.8 \%(60 / 208)$ \\
\hline Death dates differ by $>1$ month & $43.8 \%(7 / 16)$ & $5.3 \%(2 / 38)$ & $14.9 \%(23 / 154)$ & $15.4 \%(32 / 208)$ \\
\hline Death reported in ART register prior to non-death outcome in DR-TB register & $0.0 \%(0)$ & $0.3 \%(1)$ & $1.5 \%(22)$ & $1.2 \%(23)$ \\
\hline Death reported in DR-TB register prior to non-death outcome in ART register & $0.0 \%(0)$ & $0.9 \%(3)$ & $3.4 \%(49)$ & $2.7 \%(52)$ \\
\hline $\begin{array}{l}\text { Lost to follow-up in ART register, death recorded in DR-TB register within } 3 \\
\text { months }\end{array}$ & $0.0 \%(0)$ & $1.8 \%(6)$ & $4.6 \%(66)$ & $3.7 \%(72)$ \\
\hline $\begin{array}{l}\text { Lost to follow-up in DR-TB register, death recorded in ART register within } 3 \\
\text { months }\end{array}$ & $2.0 \%(3)$ & $0.9 \%(3)$ & $0.5 \%(7)$ & $0.7 \%(13)$ \\
\hline Transferred out in ART register, death recorded in DR-TB register within 3 months & $0.7 \%(1)$ & $0.3 \%(1)$ & $0.4 \%(5)$ & $0.4 \%(7)$ \\
\hline Transferred out in DR-TB register, death recorded in ART register within 3 months & $0.0 \%(0)$ & $0.0 \%(0)$ & $0.0 \%(0)$ & $0.0 \%(0)$ \\
\hline
\end{tabular}

Abbreviations: DR-TB Drug resistant tuberculosis, ART Antiretroviral therapy, FIS Focal Point Information System, TE TherapyEdge-HIV ${ }^{T M}$, EDRWeb Electronic Drug Register Web, TIER.NET The Three Integrated Electronic Registers

agreement between reporting of death was good between the DR-TB and ART registers (Kappa $=0.60)$.

\section{Discussion}

The electronic DR-TB and ART registers, used by majority of public healthcare facilities across South Africa, are crucial for monitoring treatment programme outcomes and have improved the quality of the reporting to the South African $\mathrm{NDOH}$ and international organizations such as the World Health Organization. They have also allowed for data access by various research organizations. However, they do not come without their weaknesses. Our analysis has shown an underreporting of crucial indices of patient outcomes, in particular ART start dates and death outcomes. Close to $11 \%$ of patients had a death reported in the DR-TB register but not the ART register, or vice versa. Majority of the corresponding non-death outcomes were reported as lost to follow-up, thus we can assume the patient was marked as such when they failed to return to the facility. Though these are likely explained by the design or timing of data entry in either system, it still remains problematic to researchers and government who analyze these individual data systems at face value for monitoring purposes; additional follow-up is required to ensure that death does not go underreported.
A possible solution to confirming death outcomes would be to have the electronic registers linked to the South African national death registry, however this will depend on having a valid South African identity number correctly recorded, and with the large patient numbers involved, it would need to be an automated and continuous procedure. We also found poor quality of recording of ART information in EDRWeb. The majority of patients at the two study sites that used EDRWeb exclusively did not have ART start date recorded (99.7 and 80.5\%, respectively). Though FIS also had missing ART information, it was on a smaller scale compared to EDRWeb. ART start dates and ART regimen are important to know for monitoring of the DR-TB programme, as well as monitoring drug interactions as new drugs are being introduced, for example dolutegravir for ART and bedaquiline for TB. Rates of DR-TB diagnoses in the ART programme are also underreported (as with Site 2 where just under half of patients did not have DR-TB status recorded in the ART register). Importantly, these discrepancies existed across sites and different systems, which may suggest that this could be a problem for other similar healthcare facilities across South Africa.

Our matching algorithm performed reasonably well in linking patients between datasets, however it is limited 
by the quality of the reporting of patient identifiers, and the sensitivity of the algorithm could be improved if the patient identifiers were accurately recorded in both registers. This highlights the need for a unique patient identifier within the public health system, something planned for in the National Health Insurance Policy proposed by the South African government [14].

\section{Conclusions}

The quality and completeness of the electronic DR-TB and ART registers is concerning for researchers and government who use it to monitor the outcomes of the South African TB and HIV programmes. Although South Africa's public healthcare system is understaffed and overburdened and regular internal auditing of data may not be feasible, urgent attention is needed to address data gaps in the ART and DR-TB electronic systems.

In 2016, the NDOH implemented a TB module into TIER.NET aiming to integrate the TB and HIV data to increase reporting efficiency. While this TB module can capture DR-TB through drug sensitivity and TB regimen variables, EDRWeb will still be used to monitor DR-TB patients. The impact of the TIER.NET TB module is yet to be determined, and while data integration can improve the quality of these data systems, accurate reporting is still crucial to ensure good data quality and their ability to have a meaningful impact on monitoring, policy, planning and epidemiological analysis. Furthermore, it is essential to have intensive, ongoing training of staff who capture data into electronic registers, constant emphasis on data quality, improvement of patient file management, and the transfer of information to the data capturing team.

\section{Abbreviations}

ART: Antiretroviral therapy; DR-TB: Drug resistant tuberculosis: EDRWeb: Electronic Drug Register Web; ETR.Net: Electronic TB register; FIS: Focal Point Information System; HIV: Human immunodeficiency virus; IQR: Interquartile range; MDR: Multi-drug resistant; NDOH: National Department of Health; NGO: Non-Governmental Organization; NPV: Negative predictive value; PPV: Positive predictive value; RR: Rifampicin-resistant; TB: Tuberculosis; TE: TherapyEdge-HIV TM, TIER.NET: The Three Integrated Electronic Registers

\section{Acknowledgements}

Special thanks to Dr. Nalini Singh, Dr. Iqbal Master, Dr. Nompumelelo Mthupha, Sr Loretta van der Broeck, Ms. Lebogang Ngolele and Ms. Melda Musina.

\section{Authors' contributions \\ $\mathrm{L}$ conducted the analysis and prepared the manuscript. DE, RB, SA, KW and $\mathrm{LL}$ contributed towards the analytical thinking and design, while $D E, R B$, and NI provided advice and clinical input. All authors contributed to the interpretation of data and reviewed the manuscript. All authors read and approved the final manuscript.}

\section{Funding}

This study has been made possible by the generous support of the American People and the President's Emergency Plan for AIDS Relief (PEPFAR) through US Agency for International Development (USAID) under the terms of Cooperative Agreements AID-674-A ${ }^{-12}-00029$ and
72067419CA00004 to Health Economics and Epidemiology Research Office. The contents are the responsibility of the Health Economics and Epidemiology Research Office, a Division of the Wits Health Consortium (Pty) Ltd. and do not necessarily reflect the views of USAID or the United States Government. The funders had no role in study design, data collection and analysis, decision to publish, or preparation of the manuscript.

\section{Availability of data and materials}

The data is owned by the study site and National Department of Health (South Africa) and governed by the Human Research Ethics Committee (University of Witwatersrand, Johannesburg, South Africa). All relevant data is included in the paper and supplementary tables. The full data are available from the Health Economics and Epidemiology Research Office for researchers who meet the criteria for access to confidential data and have approval from the owners of the data (information@heroza.org).

\section{Ethics approval and consent to participate}

Ethical approval was provided by the Human Research Ethics Committee (Medical) of the University of the Witwatersrand (M160506). The study protocol was reviewed by the Institutional Review Board of the University of Witwatersrand who approved the data collection without informed consent.

\section{Consent for publication}

Not applicable.

\section{Competing interests}

The authors declare that they have no competing interests.

\section{Author details}

${ }^{1}$ Health Economics and Epidemiology Research Office (HE2RO), Department of Internal Medicine, School of Clinical Medicine, Faculty of Health Sciences, University of the Witwatersrand, Johannesburg, South Africa. ${ }^{2}$ Department of Global Health, Boston University School of Public Health, Boston, USA.

${ }^{3}$ Centre for Tuberculosis, National Institute of Communicable Diseases (NICD), Johannesburg, South Africa. ${ }^{4}$ Department of Medical Microbiology, University of Pretoria, Pretoria, South Africa. ${ }^{5}$ Tuberculosis \& HIV Investigative Network (THINK), Doris Goodwin TB Hospital in Pietermaritzburg, Pietermaritzburg, South Africa.

Received: 5 September 2019 Accepted: 18 November 2019 Recerved. 5 september 2019 Accepted. 18 sons

\section{References}

1. UNAIDS. Country factsheet: South Africa. 2017. http://www.unaids.org/en/ regionscountries/countries/southafrica.

2. WHO. Global tuberculosis report. 2018. http://www.who.int/tb/publications/ global_report/en/.

3. Osler M, Hilderbrand K, Hennessey C, Arendse J, Goemaere E, Ford N, et al. A three-tier framework for monitoring antiretroviral therapy in high HIV burden settings. J Int AIDS Soc. 2014;17:18908.

4. White C. An evaluation of the implementation of the 3-tiered ART monitoring system in South Africa (Masters Thesis). University of Cape Town; 2016. https://open.uct.ac.za/handle/11427/22825.

5. du Preez K, Schaaf HS, Dunbar R, Swartz A, Bissell K, Enarson DA, et al. Incomplete registration and reporting of culture-confirmed childhood tuberculosis diagnosed in hospital. Public Health Action. 2011;1(1):19-24.

6. Mlotshwa M, Smit S, Williams S, Reddy C, Medina-Marino A. Evaluating the electronic tuberculosis register surveillance system in Eden District, Western cape, South Africa, 2015. Glob Health Action. 2017:10(1):1360560.

7. Podewils LJ, Bantubani N, Bristow C, Bronner LE, Peters A, Pym A, et al. Completeness and reliability of the Republic of South Africa National Tuberculosis (TB) surveillance system. BMC Public Health. 2015;15:765.

8. Rose PC, Schaaf HS, du Preez K, Seddon JA, Garcia-Prats AJ, Zimri K, et al. Completeness and accuracy of electronic recording of paediatric drugresistant tuberculosis in Cape Town. South Africa Public Health Action. 2013; 3(3):214-9.

9. Bristow CC, Dilraj A, Margot B, Podewils $\amalg$. Lack of patient registration in the electronic TB register for sputum smear-positive patients in KwaZuluNatal, South Africa. Tuberculosis (Edinb). 2013;93(5):567-8.

10. Levenshtein VI. Binary codes capable of correcting deletions, insertions, and reversals. Soviet Physics Doklady. 1966;10(8):707-10. 
11. SAS Institute Inc. (2018). "COMPGED Function." Accessed 1 Sept 2018. http:// support.sas.com/documentation/cdl/en/lrdict/64316/HTML/default/viewer. htm\#a002206133.htm.

12. Cadieux R, Bretheim DR. Matching Rules: Too Loose, Too Tight, or Just Right? SAS Global Forum 2014, Washington DC, March 23-26, 2014; 2014. p. 1674-2014.

13. Salas SB, Varga A, Shuster E. Fun with Address Matching: Use of the COMPGED Function and the SQL Procedure. SAS Global Forum 2018, April 8-11, 2018 - Denver, Colorado; 2018. p. 2487-018.

14. South African Department of Health National Health Insurance Policy. 2017. http://www.health.gov.za/index.php/nhi?download=2257: white-paper-nhi-2017.

\section{Publisher's Note}

Springer Nature remains neutral with regard to jurisdictional claims in published maps and institutional affiliations.

Ready to submit your research? Choose BMC and benefit from:

- fast, convenient online submission

- thorough peer review by experienced researchers in your field

- rapid publication on acceptance

- support for research data, including large and complex data types

- gold Open Access which fosters wider collaboration and increased citations

- maximum visibility for your research: over $100 \mathrm{M}$ website views per year

At BMC, research is always in progress.

Learn more biomedcentral.com/submissions 